\title{
Joachim Brinckmann
}

Über die kleinste separative Kongruenz auf einer Halbgruppe

Czechoslovak Mathematical Journal, Vol. 35 (1985), No. 2, 300-311

Persistent URL: http://dml.cz/dmlcz/102018

\section{Terms of use:}

(C) Institute of Mathematics AS CR, 1985

Institute of Mathematics of the Czech Academy of Sciences provides access to digitized documents strictly for personal use. Each copy of any part of this document must contain these Terms of use.

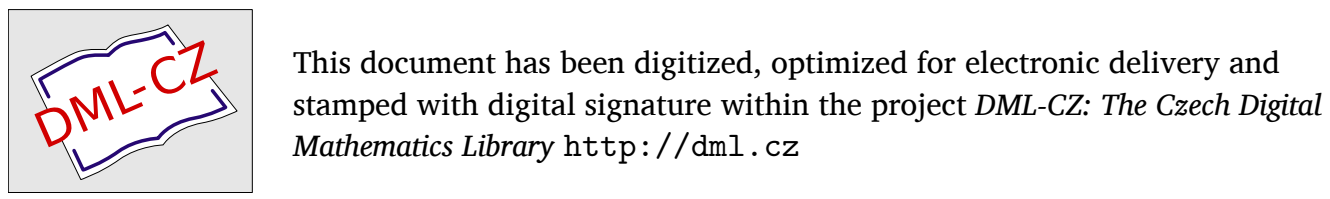




\title{
ÜBER DIE KLEINSTE SEPARATIVE KONGRUENZ AUF EINER HALBGRUPPE
}

\author{
JoAChim Brinckmann, Rostock
}

(Eingegangen am 23. Dezember 1983)

\section{EINLEITUNG}

Wir nennen eine Halbgruppe $S$ separativ, wenn aus $x^{2}=x y=y^{2}$ stets $x=y$ folgt. Eine Halbgruppe heißt linksseparativ (rechtsseparativ), wenn aus $x^{2}=x y$ und $y^{2}=y x\left(x^{2}=y x\right.$ und $\left.y^{2}=x y\right)$ stets $x=y$ folgt. Ist die Faktorstruktur $S / R$, wobei $R$ eine auf der Halbgruppe $S$ definierte Kongruenz ist, separativ (linksseparativ bzw. rechtsseparativ), so nennen wir die Kongruenz $R$ separativ (linksseparativ bzw. rechtsseparativ). Eine Kongruenz $R$ heißt kleinste separative Kongruenz auf einer Halbgruppe $S$, wenn aus $(a, b) \in R$ stets $(a, b) \in R^{\prime}$ für jede beliebige separative Kongruenz $R^{\prime}$ auf $S$ folgt.

In [3] wird eine Relation $Q$ mit

$$
\begin{gathered}
(a, b) \in Q \Leftrightarrow \text { Es gibt eine positive ganze Zahl } n \text { mit } \\
a^{n+1}=a^{n} b \text { und } b^{n+1}=b^{n} a .
\end{gathered}
$$

definiert und gezeigt, daß $Q$ die kleinste separative Kongruenz auf einer kommutativen Halbgruppe ist. Chrislock löste dieses Problem in [2] für mediale Halbgruppen. N. P. Mukherjee definierte in [6] eine Relation $Q^{*}$ mit

$$
\begin{gathered}
(a, b) \in Q^{*} \Leftrightarrow \text { Es gibt eine positive ganze Zahl } n \text { mit } \\
a^{n+1}=b a^{n} \text { und } b^{n+1}=a b^{n} .
\end{gathered}
$$

und zeigte, daß $Q^{*}$ auf einer quasikommutativen Halbgruppe die kleinste separative Kongruenz ist. Mit der Relation $R_{1}$ :

$$
\begin{gathered}
(a, b) \in R_{1} \Leftrightarrow \text { Es gibt eine positive ganze Zahl } n \text { mit } \\
a^{n} b=a^{n+1}=b a^{n} \quad \text { und } a b^{n}=b^{n+1}=b^{n} a .
\end{gathered}
$$

wurde das gleiche Problem für Duo-Halbgruppen von B. Pondělíček [9] gelöst. Fast zur gleichen Zeit zeigte R. Strecker [13], daß die Relation $R_{1}$ die kleinste separative Kongruenz auf einer $x$-kommutativen Halbgruppe ist. A. Nagy zeigte, daß 
die Relation $R_{2}$ :

$$
\begin{gathered}
(a, b) \in R_{2} \Leftrightarrow \text { Es gibt eine positive ganze Zahl } n \text { mit } \\
a b^{n}=b^{n+1}=a^{n+1}=b a^{n} .
\end{gathered}
$$

in einer vollständig symmetrischen Halbgruppe [7] und die Relation $R_{1}$ in einer schwach-kommutativen Halbgruppe [8] die kleinste separative Kongruenz ist. In [1] wurde dieses Problem vom Autor allgemeiner behandelt und auf potenzverbundene Halbgruppen angewendet. Die kleinste separative Kongruenz $R$ ist in diesem Fall:

$$
(a, b) \in R \Leftrightarrow \text { Es gibt eine positive ganze Zahl } n \text { mit }
$$

$$
a^{n}=b^{n} \quad \text { und } \quad a^{n+1}=b^{n+1} .
$$

Beziehungen zwischen den Relationen $Q, Q^{*}, R_{1}, R_{2}, R$ und anderen Relationen sowie einige Eigenschaften dieser Relationen werden im Abschnitt II behandelt. Im Abschnitt III werden wir die grundlegenden Sätze dieser Arbeit beweisen, daß z.B. die Relation $Q$ kleiner als jede andere linksseparative Kongruenz auf einer beliebigen Halbgruppe $S$ ist. Indem wir im Abschnitt IV das Radikal einer Halbgruppe einführen, leiten wir aus den Sätzen des Abschnittes III notwendige und hinreichende Kriterien dafür her, daß z. B. die Relation $Q$ die kleinste linksseparative Kongruenz auf einer Halbgruppe ist. Folgerungen und Anwendungen hieraus werden im Abschnitt $\mathrm{V}$ abgehandelt.

\section{SEPARATIVE ÄQUIVALENZRELATIONEN}

Wir behalten im folgenden die Bezeichnungen für die in der Einführung aufgelisteten Relationen bei. Die Abkürzung , $\bigvee n \in N^{+}:$“ wird für die Aussage „,Es existiert eine positive ganze Zahl $n$ mit:“" stehen.

Satz 2.1. Es sind folgende Aussagen äquivalent:

(i): $(a, b) \in R$.

(ii): $\bigvee n \in N^{+}: a^{n+1}=b^{n+1}=a^{n} b$.

(iii): $\vee n \in N^{+}: a^{n+1}=a^{n} b=b a^{n}=b^{n+1}=b^{n} a=a b^{n}$.

(iv): $\bigvee h, k \in N^{+}: a^{h}=b^{k}$ und $a^{h+1}=b^{k+1}$.

Beweis. (i) $\Leftrightarrow$ (ii) ist klar.

(ii) $\Leftrightarrow$ (iii) folgt wegen

$$
\left.b a^{n+1}=b^{n+2}=a^{n+1} b=a^{\prime} a^{n} b\right)=a^{n+2}=a b^{n+1}=b^{n+1} a .
$$

(iii) $\Leftrightarrow$ (iv) gilt wegen

$$
a^{n+2}=b^{n+1} a=b\left(b^{n} a\right)=b^{n+2} .
$$

(iv) $\Leftrightarrow$ (i): Wegen (iv) gelten die Gleichungen

$$
a^{h}=b^{k}, \quad a^{h+1}=b^{k+1}, \quad a^{h+1}=a^{h} b, \quad b^{k+1}=b^{k} a .
$$


Hieraus folgen die Beziehungen:

$$
\begin{aligned}
& a^{h} b^{k+1}=b^{k} a^{h+1}, \\
& a^{h} b^{k+1}=\left(a^{h} b\right) b^{k}=a^{h+1} b b^{k-1}=a^{h+2} b^{k-1}=\ldots=a^{h+k+1}, \\
& b^{k} a^{h+1}=\left(b^{k} a\right) a^{h}=b^{k+1} a^{h}=\ldots=b^{h+k+1}, \\
& a^{h+k+2}=b^{h+k+1} a=b^{h+1}\left(b^{k} a\right)=b^{h+k+2} .
\end{aligned}
$$

Wegen der Gleichungen (1) bis (4) gilt $(a, b) \in R$.

Folgerung 2.1. Es gilt $R=R_{1}=R_{2}$.

Folgerung 2.2. Es gilt $Q \cap Q^{*}=R$.

Folgerung 2.3. Aus $a^{n}=b^{n}$ und $a^{n+1}=b^{n+1}$ folgt $a^{n+r}=b^{n+r}$ für alle $r \in N$.

Satz 2.2. In jeder Halbgruppe $S$ sind die Relationen $Q, Q^{*}$ und $R$ Äquivalenzrelationen.

Beweis. Wir beweisen den Satz zunächst für die Relation $Q$. Reflexivität und Symmetrie von $Q$ sind klar. Die Transitivität von $Q$ folgt aus: Gelte $(a, b) \in Q$ und $(b, c) \in Q$. Dann existieren positive ganze Zahlen $m$ und $n$ mit

$$
a^{n+1}=a^{n} b, \quad b^{n+1}=b^{n} a, \quad c^{m+1}=c^{m} b, \quad b^{m+1}=b^{m} c .
$$

Ohne die Allgemeinheit zu beschränken, können wir $n=m$ setzen. Damit folgt aus den Gleichungen (5)

$$
\begin{gathered}
a^{2 n+1}=a^{n} a^{n+1}=a^{n} a^{n} b=a^{n-1} a^{n+1} b=a^{n-1} a^{n} b^{2}=\ldots=a^{n} b^{n+1}= \\
=a^{n} b^{n} c=a^{n} b b^{n-1} c=a^{n+1} b^{n-1} c=\ldots=a^{2 n} c .
\end{gathered}
$$

und

$$
c^{2 n+1}=c^{2 n} b=\ldots=c^{n} b^{n+1}=c^{n} b^{n} a=\ldots=c^{2 n} a .
$$

Also gilt $(a, c) \in Q$. Die Transitivität von $Q^{*}$ folgt analog. Wegen Folgerung 2.2 ist dann auch $R$ transitiv.

Definition 1. Wir nennen eine Relation $\varrho$ separativ (linksseparativ bzw. rechtsseparativ), wenn aus $a^{2} \varrho a b \varrho b^{2} \cdot\left(a^{2} \varrho a b\right.$ und $b^{2} \varrho b a$ bzw. $a^{2} \varrho b a$ und $\left.b^{2} \varrho a b\right)$ stets $a \varrho b$ folgt.

Satz 2.3. $R$ is eine separative Äquivalenzrelation.

Beweis. Gelte $a^{2} R a b R b^{2}$. Dann folgt aus $a^{2} R b^{2}$

$$
\bigvee n \in N^{+}: a^{2 n}=b^{2 n} \text { und } a^{2 n+2}=b^{2 n+2} \text {, }
$$

und aus $a^{2} R a b$

$$
\bigvee m \in N^{+}: a^{2 m}=(a b)^{m} \text { und } a^{2 m+2}=(a b)^{m+1}
$$


Wegen Folgerung 2.3 erhalten wir damit

$$
b^{2 n+2}=a^{2 n+2}=(a b)^{n+1}=(a b)^{n} a b=a^{2 n+1} b .
$$

Wegen Satz 2.1 erhalten wir damit $(a, b) \in R$.

Satz 2.4. Die Äquivalenzrelation $Q$ ist separativ und linksseparativ.

Beweis. Da aus $a^{2} Q a b$ und $b^{2} Q b a$ sofort $a Q b$ folgt, ist $Q$ linksseparativ. Wir zeigen nun, daß $Q$ auch separativ ist. Aus $a^{2} Q a b Q b^{2}$ folgen die Gleichungen

$$
a^{2 n+2}=a^{2 n+1} b \quad \text { und } \quad b^{2 n+2}=b^{2 n} a^{2}
$$

für ein $n \in N^{+}$. Damit gilt

$$
\begin{aligned}
b^{4 n+2}= & b^{2 n} b^{2 n+2}=b^{2 n} b^{2 n} a^{2}=b^{2 n-2} b^{2 n+2} a^{2}= \\
& =b^{2 n-2} b^{2 n} a^{4}=\ldots=b^{2 n} a^{2 n+2} .
\end{aligned}
$$

Damit folgen die Rechenschritte

$$
\begin{gathered}
b^{4 n+3}=b^{2 n} a^{2 n+2} b=b^{2 n} a^{2 n+3}=\left(b^{2 n} a^{2}\right) a^{2 n} a=b^{2 n+2} a^{2 n} a= \\
=b^{2}\left(b^{2 n} a^{2}\right) a^{2 n-2} a=b^{2 n+4} a^{2 n-2} a=\ldots=b^{4 n+2} a .
\end{gathered}
$$

Also gilt $(a, b) \in Q$.

Den folgenden Satz mit der dualen Relation $Q^{*}$ kann man analog beweisen.

Satz 2.5. Die Äquivalenzrelation $Q^{*}$ ist separativ und rechtsseparativ.

J. R. Chrislock bestimmt in der Arbeit [2] auch die kleinste Kongruenz, die sowohl links- als auch rechtsseparativ ist. Wir greifen hier diesen Gedanken auf.

Definition 2. $(a, b) \in T \Leftrightarrow$ Es existieren positive ganze Zahlen $h, k$ mit $a^{h+k+1}=$ $=a^{h} b a^{k}$ und $b^{h+k+1}=b^{h} a b^{k}$.

Folgerung 2.4. Folgende Aussagen sind äquivalent:

(i): $(a, b) \in T$.

(ii): $\bigvee n \in N^{+}: a^{2 n+1}=a^{n} b a^{n}$ und $b^{2 n+1}=b^{n} a b^{n}$.

(iii): $\bigvee h, k, n \in N^{+}: a^{h+k+1}=a^{h} b a^{k}$ und $b^{n+m+1}=b^{m} a b^{n}$.

Beweis. Aus (i) folgt (ii) durch Multiplikation mit einer geeigneten Potenz von $a$. (ii) $\Leftrightarrow$ (iii) ist trivial. (iii) $\Leftrightarrow$ (i): Sei $i=\max (h, n)$ und $j=\max (k, m)$, dann gilt

$$
a^{i+j+1}=a^{i} b a^{j} \text { und } b^{i+j+1}=b^{i} a b^{i} .
$$

Satz 2.6. Die Relation $T$ ist separativ, linksseparativ und rechtsseparativ.

Beweis. Aus $x^{2} T x y$ und $y^{2} T y x$ folgt nach (ii) aus Folgerung 2.4:

$$
x^{4 m+2}=x^{2 m+1} y x^{2 m} \text { und } y^{4 n+2}=y^{2 n+1} x y^{2 n}
$$

mit $m, n \in N^{+}$. Nach (iii) aus Folgerung 2.4 gilt damit $(a, b) \in T$. Der Nachweis der Rechtsseparativität ist analog. $\mathrm{Zu}$ zeigen bleibt die Separativität von $T$. Sei 
$x^{2} T x y T y^{2}$. Dann gilt: Es existieren $n, m \in N^{+}$mit

$$
x^{4 n+2}=x^{2 n+1} y x^{2 n} \text { und } y^{4 m+2}=y^{2 m} x y^{2 m+1} .
$$

Also ist $T$ separativ.

\section{SEPARATIVE KONGRUENZEN}

In diesem Abschnitt werden die im vorhergehenden Abschnitt betrachteten Äquivalenzrelationen in Beziehung mit links-, rechts- bzw. separativen Kongruenzen auf einer beliebigen Halbgruppe gesetzt.

Bemerkung 1. Jede linksseparative (rechtsseparative) Kongruenz $\varrho$ auf einer Halbgruppe $S$ ist separativ. Damit ist insbesondere jede linksseparative Halbgruppe auch separativ.

Beweis. Gelte $a^{2} \varrho a b \varrho b^{2}$ in $S$. Daraus folgen die Beziehungen

und

$$
a^{4} \varrho(a b)^{2} \varrho a b^{2} a=a b b a
$$

$$
(b a)^{2}=b a b a \varrho b a^{3} \varrho b a a b .
$$

Wegen der Linksseparativität von $\varrho$ folgt hieraus $a b \varrho b a$. Damit gilt $a^{2} \varrho a b$ und $b^{2} \varrho b a$, woraus wir $a \varrho b$ erhalten.

Satz 3.1. Für eine beliebige separative Kongruenz $\varrho$ folgt aus $a^{n} \varrho b^{n}$ und $a^{n+1} \varrho b^{n+1}$ mit $n \in N^{+}$die Beziehung a $\varrho b$.

Beweis. Wir zeigen, daß aus

$$
a b^{n} \varrho b^{n} a \varrho a^{n+1} \varrho b^{n+1}
$$

die Beziehung

$$
a b^{n-1} \varrho b^{n-1} a \varrho a^{n} \varrho b^{n}
$$

folgt. Wegen (1) gilt

$$
\left(b a b^{n-1}\right)^{2}=b a\left(b^{n} a\right) b^{n-1} \varrho\left(b a b^{n-1}\right) b^{n+1} \varrho\left(b^{n+1}\right)^{2} .
$$

Hieraus folgt wegen der Separativität von $\varrho$

$$
b a b^{n-1} \varrho b^{n+1} .
$$

Mit den Beziehungen (3) und (1) erhalten wir für $n \geqq 2$

Also gilt

$$
\left(a b^{n-1}\right)^{2}=a b^{n-2}\left(b a b^{n-1}\right) \varrho\left(a b^{n-1}\right) b^{n} \varrho\left(b^{n}\right)^{2} .
$$

$$
a b^{n-1} \varrho b^{n} \text {. }
$$

Mittels (3) und (1) folgt

$$
\begin{gathered}
\left(b^{n-1} a b\right)^{2}=b^{n-1} a b^{n} a b=b^{n-2}\left(b a b^{n-1}\right) b a b \varrho b^{2 n} a b \varrho\left(b^{n+1}\right)^{2}= \\
=b^{n-1} b^{n+1} b^{2} \varrho\left(b^{n-1} a b\right) b^{n+1} .
\end{gathered}
$$


Also ist $b^{n-1} a b \varrho b^{n+1}$. Wenden wir diese Beziehung an, so erhalten wir und damit

$$
\left(b^{n-1} a\right)^{2}=\left(b^{n-1} a b\right) b^{n-2} a \varrho b^{n-1}\left(b^{n} a\right) \varrho\left(b^{n}\right)^{2} \varrho\left(b^{n-1} a\right) b^{n}
$$

$$
b^{n-1} a \varrho b^{n} .
$$

Aus (1) folgt durch Multiplikation mit $a^{n-1}$

$$
a^{2 n} \varrho a^{n} b^{n} \varrho b^{n} a^{n} \varrho b^{n} a a^{n-1} \varrho b^{n+1} a^{n-1} \varrho b\left(b^{n} a\right) a^{n-2} \varrho b^{n+2} a^{n-2} \varrho \ldots \varrho b^{2 n} \text {. }
$$

Also gilt $a^{n} \varrho b^{n}$. Aus (4), (5) und $a^{n} \varrho b^{n}$ folgt (2).

Im nächsten Schritt folgt mittels analoger Rechnung aus (2) die Beziehung

$$
a b^{n-2} \varrho b^{n-2} a \varrho a^{n-1} \varrho b^{n-1}
$$

und nach weiteren $(n-3)$-Schritten

$$
a b \varrho b a \varrho a^{2} \varrho b^{2} .
$$

Da $\varrho$ separativ ist, folgt hieraus $a \varrho b$.

Folgerung 3.1. Die separative Äquivalenzrelation $R$ ist in jeder auf der Halbgruppe $S$ definierten separativen Kongruenz enthalten.

Satz 3.2. Für eine beliebige linksseparative Kongruenz $\varrho$ folgt aus $a^{n+1} \varrho a^{n} b$ und $b^{n+1} \varrho b^{n} a$ mit $n \in N^{+}$die Beziehung $a \varrho b$.

Beweis. Mit der Voraussetzung des Satzes gilt

und

$$
\left(b a b^{n-1}\right)^{2}=b a\left(b^{n} a\right) b^{n-1} \varrho\left(b a b^{n-1}\right) b^{n+1}
$$

$$
\left(b^{n+1}\right)^{2}=b^{2} b^{n+1} b^{n-1} \varrho b^{n+1}\left(b a b^{n-1}\right) .
$$

Aus den letzten beiden Beziehungen folgt wegen der Linksseparativität von $\varrho$ :

$$
b a b^{n-1} \varrho b^{n+1} .
$$

Mittels einer analogen Rechnung erhalten wir

$$
a b a^{n-1} \varrho a^{n+1} .
$$

Für $n \geqq 2$ erhalten wir mittels (7)

und

$$
\left(a^{n-1} b\right)^{2}=a^{n-2}\left(a b a^{n-1}\right) b \varrho a^{n-1}\left(a^{n} b\right) \varrho a^{n-2} a^{n+1} a \varrho\left(a^{n-1} b\right) a^{n}
$$

$$
a^{n}\left(a^{n-1} b\right)=a^{n-1}\left(a^{n} b\right) \varrho\left(a^{n}\right)^{2} .
$$

Da $\varrho$ linksseparativ ist, folgt hieraus

$$
a^{n} \varrho a^{n-1} b .
$$

Mittels einer analogen Rechnung erhalten wir

$$
\left(b^{n-1} a\right)^{2} \varrho\left(b^{n-1} a\right) b^{n} \text { und }\left(b^{n}\right)^{2} \varrho b^{n}\left(b^{n-1} a\right)
$$


und damit

$$
b^{n} \varrho b^{n-1} a .
$$

Nach $(n-2)$-Schritten erhalten wir aus den Beziehungen (8) und (9)

und damit $a \varrho b$.

$$
a^{2} \varrho a b \text { und } b^{2} \varrho b a
$$

Folgerung 3.2. Die linksseparative Äquivalenzrelation $Q$ ist in jeder auf einer Halbgruppe $S$ definierten linksseparativen Kongruenz enthalten.

Folgerung 3.3. Die Äquivalenzrelation $R$ ist in jeder auf einer Halbgruppe $S$ definierten linksseparativen Kongruenz enthalten.

Analog zum Satz 3.2 gilt der folgende Satz.

Satz 3.3. Für eine beliebige rechtsseparative Kongruenz $\varrho$ folgt aus $a^{n+1} \varrho b a^{n}$ und $b^{n+1} \varrho a b^{n}$ mit $n \in N^{+}$stets a $\varrho b$.

Folgerung 3.4. Die rechtsseparative Äquivalenzrelation $Q^{*}$ ist in jeder auf einer Halbgruppe $S$ definierten rechtsseparativen Kongruenz enthalten.

Folgerung 3.5. Die Äquivalenzrelation $R$ ist in jeder auf einer Halbgruppe $S$ definierten rechtsseparativen Kongruenz enthalten.

Sałz 3.4. Ist $\varrho$ eine linksseparative und rechtsseparative Kongruenz, so folgt aus $b^{h+k+1} \varrho b^{h} a b^{k}$ und $a^{h+k+1} \varrho a^{h} b a^{k}$ stets $a \varrho b$.

Beweis. Wegen der Voraussetzung des Satzes gilt:

und

$$
\left(b^{h-1} a b^{k}\right)^{2}=b^{h-1} a b^{k-1}\left(b^{h} a b^{k}\right) \varrho\left(b^{h-1} a b^{k}\right) b^{h+k}
$$

$$
\left(b^{h+k}\right)^{2} \varrho\left(b^{h+k}\right) b^{h-1} a b^{k} .
$$

Wegen der Linksseparativität von $\varrho$ folgt hieraus

$$
b^{h+k} \varrho b^{h-1} a b^{k} \text {. }
$$

Analog erhalten wir

$$
a^{h+k} \varrho a^{h-1} b a^{k} .
$$

Nach $(h-2)$ analogen Rechnungen bekommen wir die Beziehungen

$$
b^{k+2} \varrho b a b^{k} \text { und } a^{k+2} \varrho a b a^{k} .
$$

Mit Hilfe dieser Beziehungen gilt

und

$$
\left(a b^{k}\right)^{2} \varrho a b^{k-1} b^{k+2}=\left(a b^{k}\right) b^{k+1}
$$

$$
b^{k+1}\left(a b^{k}\right)=b^{k}\left(b a b^{k}\right) \varrho\left(b^{k+1}\right)^{2},
$$

woraus wegen der Linksseparativität von $\varrho$

$$
b^{k+1} \varrho a b^{k}
$$


folgt. Analog folgt auch die Beziehung

$$
a^{k+1} \varrho b a^{k} .
$$

Da $\varrho$ rechtsseparativ ist, folgt aus den Beziehungen (12) und (13) wegen des Satzes 3.3 die Beziehung $a \varrho b$. Damit ist der Satz vollständig bewiesen.

Folgerung 3.6. Die linksseparative, rechtsseparative und separative Relation $T$ ist in jeder auf einer Halbgruppe $S$ definierten links- und rechtsseparativen Kongruenz enthalten.

\section{DAS RADIKAL EINER HALBGRUPPE}

Das Radikal einer Halbgruppe $S$ wurde von H.-J. Hoehnke [4] eingeführt. H. Seidel gab in seiner Arbeit [12] für das Radikal $\operatorname{rad} S$ einer Halbgruppe $S$ die folgende Charakterisierung:

Es gilt $(a, b) \in \operatorname{rad} S \Leftrightarrow \mathrm{Zu}$ jedem $s \in S$ existieren natürliche Zahlen $i, j, k, l>0$ mit

$$
(a s)^{i} a=(a s)^{j} b \text { und }(b s)^{k} a=(b s)^{l} b .
$$

In den obigen Gleichungen (1) kann nach H.-J. Hoehnke [5] $i=j=k=l$ gewählt werden.

Das Radikal rad $S$ ist in jeder Halbgruppe eine Kongruenz. Dual zum Radikal $\operatorname{rad} S$ läßt sich in jeder Halbgruppe $S$ eine Kongruenz $(\operatorname{rad} S)^{*}$ definieren:

$(a, b) \in(\operatorname{rad} S)^{*} \Leftrightarrow \mathrm{Zu}$ jedem $s \in S$ existiert eine natürliche Zahl $n>0$ mit

Dann ist auch

$$
a(s a)^{n}=b(s a)^{n} \quad \text { und } \quad a(s b)^{n}=b(s b)^{n} .
$$

$$
\overline{\operatorname{rad} S}=\operatorname{rad} S \cap(\operatorname{rad} S)^{*}
$$

eine Kongruenz in jeder Halbgruppe $S$.

Zwischen den in den vorhergehenden Abschnitten behandelten Relationen und in diesem Abschnitt definierten Kongruenzen bestehen folgende Beziehungen:

$$
\begin{gathered}
R \subseteq Q \subseteq T, \quad R \subseteq Q^{*} \subseteq T, \quad Q \supseteq \operatorname{rad} S, \\
Q^{*} \supseteq(\operatorname{rad} S)^{*}, \quad R \supseteq \overline{\operatorname{rad} S} .
\end{gathered}
$$

Satz 4.1. In einer Halbgruppe $S$ sind folgende Aussagen äquivalent:

(i): $Q$ ist linkscompatibel.

(ii): $Q$ ist compatibel.

(iii): $Q=\operatorname{rad} S$.

(iv): rad $S$ ist die kleinste linksseparative Kongruenz auf $S$.

(v): $S / \mathrm{rad} S$ ist das maximale homomorphe Bild von $S$, welches linksseparativ ist.

Beweis. Sei $(a, b) \in Q$ und $s$ ein beliebiges Element aus $S$. Dann existiert ein 
$n \in N^{+}$mit

$$
(s a)^{n+1}=(s a)^{n} s b \text { und }(s b)^{n+1}=(s b)^{n} s a,
$$

wenn $Q$ linkscompatibel ist. Aus den letzten beiden Gleichungen folgt

$$
a s(a s)^{n} a=a s(a s)^{n} b \text { und } b s(b s)^{n} b=b s(b s)^{n} a .
$$

Also gilt $(a, b) \in \operatorname{rad} S$. Deshalb ist (i) $\Leftrightarrow$ (ii) $\Leftrightarrow$ (iii). (iii) $\Rightarrow$ (iv) gilt wegen Folgerung 3.2 (iv) $\Rightarrow$ (iii) gilt wegen (2) und Folgerung 3.2. (iv) $\Leftrightarrow$ (v) gilt wegen des Prinzips vom maximalen homomorphen Bild eines gegebenen Typs [3].

Analog gilt auch die duale Aussage zu Satz 4.1:

Satz 4.2. In einer Halbgruppe $S$ sind folgende Aussagen äquivalent:

(i): $Q^{*}$ is rechtscompatibel.

(ii): $Q^{*}$ ist compatibel.

(iii): $Q^{*}=(\operatorname{rad} S)^{*}$.

(iv): $Q^{*}$ ist die kleinste rechtsseparative Kongruenz auf $S$.

$(\mathrm{v}): S /(\operatorname{rad} S)^{*}$ ist das maximale homomorphe Bild von $S$, welches rechtsseparativ ist.

Folgerung 4.1. $Q$ ist genau dann die kleinste linksseparative Kongruenz auf einer Halbgruppe $S$, wenn $Q=\operatorname{rad} S$ gilt.

Die entsprechende duale Aussage gilt auch.

Wenden wir die Folgerung 4.1 als ein Kriterium an, so brauchen wir wegen (2) nur die Beziehung $Q \subseteq \operatorname{rad} S$ nachzuprüfen.

Eine Halbgruppe $S$ heißt radikalfrei, wenn aus $(a, b) \in \operatorname{rad} S$ stets $a=b$ folgt.

Satz 4.3. Ist $S$ eine Halbgruppe, in der $Q=\operatorname{rad} S$ gilt, so sind die folgenden Aussagen äquivalent:

(i): $(a, b) \in Q$ impliziert $a=b$.

(ii): Die Halbgruppe $S$ ist linksseparativ.

(iii): $S$ ist radikalfrei.

Beweis. (i) $\Leftrightarrow$ (iii) ist trivial. (i) $\Rightarrow$ (ii): Gelten für beliebige $a, b \in S$ die Beziehungen $a^{2}=a b$ und $b^{2}=b a$, so folgt daraus $(a, b) \in Q$. Wegen (i) gilt deshalb $a=b$. Also ist $S$ linksseparativ. (ii) $\Rightarrow$ (i) folgt unmittelbar aus dem Satz 4.1.

Satz 4.4. Eine Halbgruppe $S$, in der $Q^{*}=(\operatorname{rad} S)^{*}$ gilt, ist genau dann rechtsseparativ, wenn aus $(a, b) \in Q^{*}$ stets $a=b$ folgt.

Der Beweis dieses Satzes und die Beweise der nächsten beiden Sätze verlaufen ähnlich den Beweisen entsprechend vorangegangener Sätze.

Satz 4.5. In einer Halbgruppe $S$ sind die folgenden Aussagen äquivalent:

(i): $R$ ist compatibel.

(ii): $R=\overline{\operatorname{rad} S}$. 
(iii): $R$ ist die kleinste separative Kongruenz auf $S$.

(iv): $S / R$ ist das maximale homomorphe Bild von $S$, welches separativ ist.

Satz 4.6. Eine Halbgruppe $S$, in der die Beziehung $R=\overline{\operatorname{rad} S}$ erfüllt ist, ist genau dann separativ, wenn aus $(a, b) \in R$ stets $a=b$ folgt.

Satz 4.7. In einer Halbgruppe $S$ sind die folgenden Aussagen äquivalent:

(i): $Q=\overline{\operatorname{rad} S}$.

(ii): $Q=R=\overline{\operatorname{rad} S}=\operatorname{rad} S$.

(iii): $R$ ist die kleinste separative und die kleinste linksseparative Kongruenz auf $S$.

Beweis. Mit (i) und (2) erhalten wir $Q=\overline{\operatorname{rad} S} \subseteq R \subseteq Q$, woraus $Q=\overline{\operatorname{rad} S}=$ $=R$ folgt. Wegen $\overline{\operatorname{rad} S} \subseteq \operatorname{rad} S \subseteq Q$ erhalten wir damit (ii). Aus (ii) folgt wegen Satz 4.5 die Aussage (iii). Wegen (2) und Folgerung 3.2 gilt $Q=R$. Mit Hilfe des Satzes 4.5 folgt damit $Q=\overline{\operatorname{rad} S}$.

Der hierzu duale Satz lautet:

Satz 4.8. In einer Halbgruppe $S$ sind die folgenden Aussagen äquivalent:

(i): $Q^{*}=\overline{\operatorname{rad} S}$.

(ii): $Q^{*}=\overline{\operatorname{rad} S}=\operatorname{rad} S=R$.

(iii): $R$ ist die kleinste separative und die kleinste linksseparative Kongruenz auf $S$.

Eine Folgerung aus den Sätzen 4.3 und 4.4 ist der folgende Satz.

Satz 4.9. Gilt in einer Halbgruppe $S$ die Beziehung $Q=R\left(Q^{*}=R\right)$, so sind die Aussagen

(i): $S$ ist separativ.

(ii): $S$ ist linksseparativ (rechtsseparativ). äquivalent.

Satz 4.10. In einer Halbgruppe $S$ sind die Aussagen

(i): $Q=Q^{*}=\operatorname{rad} S$.

(ii): $Q=Q^{*}=R=\operatorname{rad} S=(\operatorname{rad} S)^{*}=\overline{\operatorname{rad} S}$.

(iii): $R$ ist die kleinste separative, kleinste linksseparative und die kleinste rechtsseparative Kongruenz auf $S$.

äquivalent.

Beweis. Zunächst erinnern wir daran, daß nach Folgerung 2.2 die Beziehung $R=Q \cap Q^{*}$ gilt. Wegen Satz 4.2 folgt dann $Q^{*}=(\operatorname{rad} S)^{*}$ und damit die Aussage (ii). Aus den Sätzen 4.1, 4.2 und 4.5 folgt die Aussage (iii). Nach (2) gilt $R \subseteq Q$. Aus (iii) und Folgerung 3.2 folgt aber $Q \subseteq R$. Also ist $R=Q$. Analog folgt $R=Q^{*}$. Mit Hilfe von Satz 4.1 erhalten wir hieraus die Aussage (i).

Folgerung 4.2. Ist in einer Halbgruppe $S$ die Relation $Q$ die kleinste linkssepara- 
tive Kongruenz und die Relation $Q^{*}$ die kleinste rechtsseparative Kongruenz auf $S$, so ist die Relation $R$ die kleinste separative Kongruenz auf $S$.

Beweis. Aus $Q=\operatorname{rad} S$ und $Q^{*}=(\operatorname{rad} S)^{*}$ folgt $R=\overline{\operatorname{rad} S}$.

Folgerung 4.3. Gilt in einer Halbgruppe $S$ die Beziehung $Q=Q^{*}=\operatorname{rad} S$, so sind die folgenden Aussagen äquivalent:

(i): $S$ ist separativ.

(ii): $S$ ist linksseparativ.

(iii): $S$ ist rechtsseparativ.

Wir nennen eine Halbgruppe $S$ radikal, wenn für alle Paare $(a, b)$ mit $a, b \in S$ gilt: $(a, b) \in \operatorname{rad} S$.

Satz 4.11. Gilt in einer Halbgruppe $S$ die Beziehung $Q=Q^{*}=\operatorname{rad} S$, so ist die Halbgruppe genau dann radikal, wenn $S$ nilpotent ist.

Beweis. Aus $Q=Q^{*}=\operatorname{rad} S$ folgt, daß $R=\operatorname{rad} S$ gilt. Ist $S$ radikal, so ist $S$ potenzverbunden. (Eine Halbgruppe $S$ heißt potenzverbunden, wenn für beliebige $a, b \in S$ natürliche Zahlen $h$ und $k$ existieren mit $a^{h}=b^{k}$.) Nach [1] gilt, daß eine potenzverbundene Halbgruppe genau dann radikal ist, wenn sie nilpotent ist. Andererseits ist jede nilpotente Halbgruppe radikal [10].

\section{ANWENDUNGEN}

$1^{\circ}$. Die in der Einleitung dieser Arbeit zitierten Beispiele lassen sich mit Hilfe der Sätze aus dem Abschnitt IV leicht überprüfen bzw. ergänzen (s. z.B. $3^{\circ}$ ).

$2^{\circ}$. Anwendung auf eine Exponentialhalbgruppe

Definition 5.1. Eine Halbgruppe $S$ heißt exponential, wenn für alle $a, b \in S$ und alle $n \in N$ gilt: $(a b)^{n}=a^{n} b^{n}$.

Man erkennt sofort, daß die Relation $R$ nach Satz 2.1 (iv) compatibel ist. Wegen Satz 4.5 folgt deshalb

Satz 5.1. In einer Exponentialhalbgruppe $S$ ist $R$ die kleinste separative Kongruenz auf $S$.

Folgerung 5.1. In einer separativen Exponentialhalbgruppe $S$ folgt aus $(a, b) \in R$ stets $a=b$.

$3^{\circ}$ : Rechts (links) schwach-kommutative Halbgruppen

Definition 5.2. Eine Halbgruppe $S$ wird rechts (links) schwach-kommutativ genannt, wenn für alle $a, b \in S$ ein $x \in S$ und ein $k \in N^{+}$existieren, so daß $(a b)^{k}=x a$ $\left((a b)^{k}=b x\right)$ gilt $[11]$.

Hilfssatz 5.1. Ist $S$ eine rechts schwach-kommutative Halbgruppe und $a, b \in S$, so existieren zu jedem $n \in N^{+}$ein $x \in S$ und ein $m \in N^{+}$mit $(a b)^{m}=x a^{n}$. 
Der Beweis dieses Satzes findet man für links schwach-kommutative Halbgruppen bei B. Pondělíček [9]. Für rechts schwach-kommutative Halbgruppen verläuft der Beweis analog.

Satz 5.2. In einer rechts schwach-kommutativen Halbgruppe $S$ ist $Q$ die kleinste linksseparative Kongruenz auf $S$.

Beweis. Wir zeigen $Q \subseteq \operatorname{rad} S$. Aus $(a, b) \in Q$ folgt: $\bigvee n \in N^{+}: a^{n+1}=a^{n} b$ und $b^{n+1}=b^{n} a$. Wegen Hilfssatz 5.1 gilt: $\mathrm{Zu}$ jedem $a \in S$, zu jedem $s \in S$ und zu beliebigen $n \in N^{+}$existieren $x \in S$ und $m \in N^{+}$mit $(a s)^{m}=x a^{n}$. Also gilt

und analog erhält man

$$
x a^{n} a=(a s)^{m} a=x a^{n} b=(a s)^{m} b
$$

$$
(b s)^{i} a=(b s)^{i} b
$$

für ein $i \in N^{+}$. Wegen Folgerung 4.1 ist damit dieser Satz bewiesen. Analog beweist man den

Satz 5.3. In einer links schwach-kommutativen Halbgruppe $S$ ist $Q^{*}$ die kleinste rechtsseparative Kongruenz auf $S$.

Definition 5.2. Eine Halbgruppe $S$ heißt schwach-kommutativ, wenn für alle $a, b \in S$ ein $n \in N^{+}$und $x, y \in S$ mit $(a b)^{k}=x a=b y$ existieren.

Folgerung 5.2. In einer schwach-kommutativen Halbgruppe ist $Q$ die kleinste rechtsseparative, $Q^{*}$ die kleinste linksseparative und $R$ die kleinste separative Kongruenz auf $S$.

Beweis. Anwendung der Sätze 5.2, 5.3 und Folgerung 4.2.

\section{Literatur}

[1] Brinckmann, J.: Das Radikal in potenzverbundenen Halbgruppen, Beiträge zur Geometrie und Algebra, 18 (1984), 49-61.

[2] Chrislock, J. L.: On medial semigroups, J. Algebra 12, 1969.

[3] Clifford, A. H. and Preston, G. B.: The Algebraic Theory of Semigroups, Amer. Math. Soc., Providence, 1961.

[4] Hoehnke, H.-J.: Structure of semigroups. Cannad. J. Math. 18, 1965.

[5] Hoehnke, H.-J.: Über das untere und obere Radikal einer Halbgruppe. Math. Z. 89, 1965.

[6] Mukherjee, N. P.: Quasi commutative semigroups I, Czech. Math. J. 22, 1972.

[7] Nagy, A.: The Least Separative Congruence on a Completely Symmetrical Semigroup, K. Marx Univ. Economics, Dept. Math., Budapest, 1980-1984.

[8] Nagy, A.: The Least Separative Congruence on a Weakly Commutative Semigroup, Czech. Math. J. 32, 1982.

[9] Ponděliček, B.: On weakly commutative semigroups, Czech. Math. J. 25, 1975.

[10] Roiz, E. N. und Schein, B. M.: Radicals of semigroups, Semigroup Forum, 1978.

[11] Sedlock, J. T.: GREEN's relation on a periodic semigroup, Czech. Math. J. 19, 1969.

[12] Seidel, H.: Über das Radikal einer Halbgruppe. Math. Nachr. 29 (1965).

[13] Strecker, R.: Über das Radikal $\chi$-kommutativer Halbgruppen. Math. Nachr. 68, 1975.

Anschrift des Verfassers: 2500 Rostock 6, Max-Planck-Strasse 14, DDR. 